\title{
Le Conseil de l'Europe et les langues de scolarisation
}

The Council of Europe and languages of education

El Consejo de Europa y las lenguas de escolarización

Jean-Claude Beacco

\section{(2) OpenEdition}

\section{Journals}

Édition électronique

URL : https://journals.openedition.org/ries/4521

DOI : 10.4000/ries.4521

ISSN : 2261-4265

Éditeur

France Education international

Édition imprimée

Date de publication : 1 décembre 2015

Pagination : 147-157

ISSN : $1254-4590$

Référence électronique

Jean-Claude Beacco, "Le Conseil de l'Europe et les langues de scolarisation», Revue internationale d'éducation de Sèvres [En ligne], 70 | décembre 2015, mis en ligne le 01 décembre 2017, consulté le 01 juillet 2021. URL : http://journals.openedition.org/ries/4521 ; DOI : https://doi.org/10.4000/ries.4521

(c) Tous droits réservés 


\title{
Le Conseil de l'Europe et les langues de scolarisation
}

\author{
Jean-Claude Beacco \\ Université Paris III Sorbonne nouvelle
}

La question de la/des langue(s) de scolarisation se présente sous deux aspects : ceux-ci peuvent être techniques/didactiques, c'est-à-dire renvoyer à des choix structuraux relatifs aux programmes d'enseignement (finalités, objectifs, contenus...) et aux modalités de leur mise en œuvre, avec des questions telles que : comment sont définies les compétences attendues dans la langue nationale/ régionale ? Quelle place pour la littérature nationale/régionale dans la scolarité obligatoire ?... Ces questions peuvent alors être dites "politiques » au sens au sens de l'anglais policy. Mais, de manière plus visible, la détermination d'une langue ou de plusieurs pour l'École relève de choix sociétaux (donc politiques, au sens de politics) en réponse aux débats et aux conflits que génère la lutte pour la reconnaissance des langues « non nationales » ou à statut d'officialité moindre, dans la mesure où le système éducatif constitue un espace déterminant de légitimation. En Europe, où les langues principales de scolarisation sont établies souvent de longue date, la question ne se pose qu'en cas de revendications des locuteurs de voir leur langue identitaire accéder à ce type de statut.

On considérera que la question des objectifs des programmes concerne la didactique des langues et que celle du statut juridique des langues ainsi que celle des finalités éducatives de leur enseignement concerne l'organisation du système scolaire et relève du politique. Cette distinction n'est certes pas très satisfaisante, puisque le choix d'une (nouvelle) langue de scolarisation peut avoir des incidences sur ses finalités et sur ses contenus. Mais elle cherche à montrer que les actions menées sur ces deux plans ne sont pas en relation directe ni même complémentaire : ils relèvent d'instances et de stratégies du changement qui sont, par nature, diverses et ils peuvent même être déconnectés l'un de l'autre. On illustrera ces décalages à travers le cas d'une organisation intergouvernementale comme le Conseil de l'Europe, dont l'influence sur les choix nationaux / régionaux ne peut être que limitée du fait de sa nature même et qui a donc privilégié la " coopération didactique » et la question, souvent négligée, des langues de scolarisation à travers les matières autres que la langue comme matière scolaire particulière.

Avant de chercher à caractériser son action en ce qui concerne les langues de scolarisation, il n'est pas inutile de situer le Conseil de l'Europe, souvent très mal distingué de l'Union européenne : organisation intergouvernementale créée en 1949, le Conseil de l'Europe réunit 47 États, dont 28 sont aussi 
membres de l'Union européenne. Tous ont adhéré à la Convention européenne des droits de l'homme ${ }^{1}$ qui est destinée à la protection des droits de l'homme, de la démocratie et de la prééminence du droit, les trois "piliers » du Conseil. La Cour européenne des droits de l'homme est chargée d'instruire les plaintes des citoyens des États membres pour violation des droits (tels que définis par la Convention) dans leur propre État. Une Convention culturelle européenne (1954) établit un cadre pour le développement d'une coopération entre les États membres dans les domaines de la culture et de l'éducation, dans l'esprit des valeurs fondamentales du Conseil de l'Europe : elle a, en particulier, pour finalité de développer la compréhension mutuelle entre les pays en encourageant l'étude de l'histoire et des civilisations ainsi que des langues.

\section{LES LANGUES DANS L'ENSEIGNEMENT : QUE PEUT LE CONSEIL DE L'EUROPE ?}

Les organisations internationales (comme l'Unesco) ont été amenées à prendre des positions sur les questions de langues, surtout dans la mesure où celles-ci concernent les droits individuels. Elles sont amenées à agir sur un plan autre que celui des États singuliers qui cherchent à défendre leurs intérêts propres (par exemple, à promouvoir leur langue nationale) et, de ce fait, elles sont porteuses de valeurs plus universelles. Mais cette indispensable largeur de vue supranationale se paie de certaines formes de dilution du message, puisque ces organisations ne disposent pas des mêmes moyens d'action que les États. Cependant, alors que les prises de position de ces organisations sont facilement considérées dans l'opinion publique comme utopiques ou vaines, elles sont utilisées par les acteurs sociaux sur place pour justifier de l'extérieur leurs propres positions et leur action. Ce sont eux qui mettent concrètement en œuvre ces " grands principes » et qui les traduisent en termes adaptés à leur contexte spécifique.

Le Conseil de l'Europe se trouve dans cette situation : il agit par concertation entre les États membres et par la recherche du consensus, dans le cadre de ses valeurs centrales et en partant du principe que la démocratie ne s'impose pas du dehors. De ce fait, il ne dispose pas d'instruments juridiquement contraignants, comme l'Union européenne. Ses modalités d'intervention sont des programmes intergouvernementaux qui, dans le domaine de l'enseignement et de l'apprentissage des langues, ont été coordonnés par la Division des langues vivantes (actuellement Unité des politiques linguistiques) ${ }^{2}$. Ceux-ci ont conduit à la réalisation d'instruments, dont le plus connu est le Cadre européen commun de référence pour les langues (CECR). Ce sont ces coopérations qui font

1. [http://www.coe.int/fr/web/about-us/who-we-are]

2. [http://www.coe.int/t/dg4/linguistic/default_fr.asp] 
« avancer » certaines idées, à travers des conférences intergouvernementales, des séminaires et surtout des instruments partagés. Le Conseil de l'Europe agit aussi à travers d'autres moyens plus politiques, qui ont un certain statut juridique : des conventions cadre, comme celle pour la protection des minorités nationales ${ }^{3}$, des chartes dont, pour ce qui concerne les langues, la Charte européenne des langues régionales ou minoritaires ${ }^{4}$ ou des recommandations aux États membres.

Ce n'est pas tant la diversité des contextes linguistiques qui freine l'action du Conseil que le fait que les États européens tendent à considérer que l'éducation relève de leur seule responsabilité (principe de subsidiarité de l'Union européenne). Mais surtout, créer des espaces de coopération entre les États membres conduit, en premier lieu, à se situer sur le plan juridique, celui des principes fondateurs de la démocratie : dans le cas des langues, il s'agit des droits linguistiques des minorités et, en particulier, de la place qui est faite, dans les systèmes éducatifs, aux langues sans statut officiel, dont l'absence de reconnaissance peut susciter des tensions politiques. Mais l'approche juridique conduit à sous-estimer les choix effectifs et les comportements concrets des États en matière d'éducation aux langues, laquelle se construit sur des choix techniques/didactiques qui ne relèvent certes pas du droit, comme enseigner la grammaire ou organiser l'enseignement par compétences.

Or, dans ce domaine, des actions concertées et convergentes entre les États peuvent se fonder sur des finalités éducatives partagées, qui ne sont pas à strictement parler politiques, comme l'éducation plurilingue et interculturelle, la primauté d'une éducation de qualité ${ }^{5}$. Le Conseil de l'Europe a été en mesure de traduire celles-ci en termes opérationnels, au moyen d'instruments de cadrage partagés qui surplombent les programmes nationaux et qui doivent être contextualisés par ceux-ci.

\section{LA VOIE POLITIQUE/JURIDIQUE : LES LANGUES DES MINORITÉS}

Le Conseil de l'Europe a proposé (1992) à ses membres une Charte européenne des langues régionales ou minoritaires. Celle-ci est ouverte à leur signature et non imposée et elle est "à la carte ", chaque signataire pouvant choisir un certain nombre de dispositions du texte intégral, avec un minimum (35) et une distribution obligatoire dans les trois différentes sections qui énumèrent les dispositions en faveur de ces langues ${ }^{6}$. Vingt-cinq États sont signataires de la

\footnotetext{
3. [http://www.coe.int/en/web/minorities/home]

4. [http://www.coe.int/t/dg4/education/minlang/default_fr.asp]

5. On notera que la notion de « droit à une éducation de qualité » ne peut être utilisée dans les documents produits au Conseil de l'Europe, car la Convention ne mentionne que le « droit à l'éducation ».

6. Article 2.2: «En ce qui concerne toute langue indiquée au moment de la ratification, de l'acceptation ou de l'approbation, conformément à l'article 3, chaque Partie s'engage à appliquer un minimum de trente-cinq paragraphes ou alinéas choisis parmi les dispositions de la partie III de la présente Charte, dont au moins trois choisis dans chacun des articles 8 et 12 et un dans chacun des articles 9, 10, 11 et 13. ".
} 
Charte. Celle-ci concerne un grand nombre de langues, dont les langues officielles/majoritaires dans des pays autres ou des langues régionales. La mise en œuvre de la Charte fait l'objet d'un suivi, tous les trois ans, par un comité d'experts du Conseil de l'Europe qui en évalue l'application et cherche à améliorer celle-ci. C'est un instrument spécifique aux langues régionales et minoritaires, très original dans sa conception.

Mais il reste sur le terrain juridique et n'analyse pas concrètement les programmes ou les dispositifs pédagogiques. Tant et si bien qu'il n'est pas facile d'évaluer ses effets dans des situations de conflits linguistiques internes. L'Ukraine, la Serbie et la Croatie ont ratifié la Charte, ce qui n'a pas suffit à apaiser les tensions. C'est que la Charte n'aborde pas les questions techniques des relations entre la langue de scolarisation principale avec les enseignements en langues régionales et minoritaires (langue dans les matières scolaires), pas davantage que celle de l'enseignement de ces langues comme matière, question beaucoup plus sensible sur un plan symbolique, puisqu'elle remet en cause l'idéologie répandue de l'unité linguistique de la nation. Or cet instrument et ses experts ne sont pas en mesure de gérer ces aspects de l'enseignement des langues de scolarisation. Et les relations n'ont jamais été très étroites entre les juristes de la Charte et les linguistes-didacticiens impliqués dans les activités de l'Unité des politiques linguistiques.

Celle-ci, dans sa dynamique propre, a proposé aux États membres (2002) un dispositif de dialogue et d'expertise destiné à les accompagner dans l'analyse de leur politique linguistique éducative et dans leurs projets de mise en place d'enseignements de langues tournés vers le plurilinguisme : les Profils des politiques linguistiques ${ }^{7}$. Il s'agit d'un processus de réflexion mené par les autorités compétentes, qui implique aussi la société civile et dans lequel les experts du Conseil de l'Europe ont une fonction de catalyseurs. Les Profils (dont 17 ont été organisés à ce jour) sont un processus d'auto-analyse assistée, destiné essentiellement à identifier les développements possibles et souhaitables des politiques linguistiques éducatives dans leur ensemble.

Les experts des Profils vont évidemment rencontrer la question des langues nationales/officielles, qui se posent en termes didactiques mais aussi juridiques/politiques pour ce qui est de la place faite aux autres langues du territoire. C'est ce que montre par exemple cet extrait du Profil Lituanie :

[...] cette très forte concentration sur la langue nationale peut être considérée comme une attitude ambivalente à l'égard des langues minoritaires ou étrangères. Comme dans la plupart des pays qui ont retrouvé leur indépendance récemment, la demande sociale en matière de langues étrangères, et notamment d'anglais, est très forte. Le respect et la protection des langues minoritaires sont garantis par la Constitution. Le russe reste une langue importante dans certains

7. [http://www.coe.int/t/dg4/linguistic/Profils_FR.asp] 
secteurs de l'économie, ainsi qu'en tant que deuxième langue étrangère (dans les écoles "majoritaires») ou langue de scolarisation (dans les écoles " minoritaires »). Le paysage linguistique lituanien est donc complexe. Le multilinguisme est perçu comme une réalité, une nécessité et une chance, mais aussi comme une menace potentielle ou directe pour la langue lituanienne, qui est le fondement de l'identité nationale. ${ }^{8}$

La question est bien de nature politique dans la mesure où les conflits résident, en effet, dans la volonté que la langue de sa communauté soit (la seule langue) officielle et de scolarisation. Les entités nationales ou territoriales, anciennes ou récentes (par exemple la Catalogne ou la Bosnie-Herzégovine) ont identifié leur langue de scolarisation, qui est le plus souvent celle constitutive de leur identité collective, mais cette prééminence peut être contestée et remise en cause au nom de la démocratie culturelle. Car l'homogénéité linguistique des États est un mythe et certains d'entre eux ont aménagé leur multilinguisme : certaines parties de l'espace politique peuvent disposer d'une autre langue pour l'école, reconnue par la Constitution (comme la Slovénie). Ces équilibres qui peuvent se trouver modifiés avec la volonté d'un groupe de faire accéder une langue régionale au statut de langue d'enseignement et même conduire à des partitions de fait de l'État. Partout où des formes d'enseignement bi/plurilingues sont en place (comme au Val d'Aoste ou au Luxembourg), les situations semblent relativement stabilisées. Les langues apportées par les personnes migrantes ne peuvent prétendre qu'à être "prises en compte " (didactique) dans l'éducation des enfants scolarisés, à être considérées comme une ressource pour la richesse linguistique collective et à ne pas servir de prétexte pour bloquer l'accès à la résidence ou à la citoyenneté des nouveaux venus. Sur ces questions, le Conseil de l'Europe a élaboré des instruments de cadrage ${ }^{9}$ pour les États membres, afin d'accompagner leur réflexion et leur analyse.

La tolérance globale à la diversité linguistique donne le sentiment d'avoir fait quelque progrès en Europe, d'autant que le coût politique de l'homogénéisation linguistique sous la contrainte ou du monolinguisme d'État devient trop important au regard des principes de la démocratie. Il n'est d'ailleurs pas à exclure que ces langues de minorités ne soient prises en otage par ceux qui, en les défendant, luttent en fait pour leur autonomie politique ou leur indépendance. Quoi qu'il en soit, les Profils des politiques linguistiques se situent sur un plan technique et ils ne sont pas en posture d'élaborer des recommandations autres que celles fondées sur des principes de politique linguistique communs (l'éducation plurilingue et interculturelle), sans fondement juridique, et dont les États membres font l'usage qu'ils souhaitent en fonction de leur adhésion à ceux-ci.

8. [http://goo.gl/tu8sP3]

9. [http://www.coe.int/fr/web/lang-migrants] 


\section{LA VOIE DIDACTIQUE : L'ÉDUCATION PLURILINGUE ET INTERCULTURELLE}

Son long passé de langues vivantes n'a pas particulièrement préparé le Conseil à aborder les problématiques du choix de la/des langues officielles utilisées à l'école et de leur mise en œuvre pédagogique. Mais celui-ci l'a conduit à privilégier la voie de coopération technique/didactique : c'est le concept d'éducation plurilingue et interculturelle qui a permis au Conseil de l'Europe de créer des formes de coopération entre les États membres à propos des langues de scolarisation à travers des instruments partagés.

On ne décrira pas ici les premiers projets du Conseil de l'Europe, qui ont eu pour objectif la démocratisation de l'apprentissage des langues et la conception d'instruments techniques destinés à faciliter l'acquisition de compétences de communication. On signalera cependant la création d'instruments de référence communs permettant de construire des contenus d'enseignement, " formule » qui va donner naissance aux documents-cadres et aux "guides", instruments permettant la coopération intergouvernementale sur le plan de l'ingénierie des formations en langues. Ces premiers instruments (Threshold Level pour l'anglais (1975) et Un niveau seuil (1976) pour le français) ont eu une influence considérable sur les programmes d'enseignement des langues dans les États et ils ont assuré la notoriété du Conseil de l'Europe dans les milieux des professionnels des langues.

La période des années 1980 et 1990 va connaître une double évolution : la concrétisation de la structuration des objectifs d'apprentissage des langues vivantes (par compétences et descripteurs de compétences) et surtout l'apparition de la notion de plurilinguisme, l'une et l'autre portées par le CECR. Celle-ci est déjà présente dans une Recommandation (98-6) ${ }^{10}$ concernant les langues vivantes, qui place la communication interculturelle et le plurilinguisme au rang d'objectif politique essentiel et qui occupe une place centrale dans le CECR (en cours d'élaboration depuis 1991, version provisoire 1997, publication 2001).

Le CECR a pour objet de recenser de manière articulée et analytique les paramètres les plus importants intervenant dans l'enseignement-apprentissage des langues et d'en donner une description hiérarchisée et transparentes. Mais le CECR comporte aussi une autre dimension, qui concerne les finalités des enseignements-apprentissages des langues. Celles-ci n’y sont pas très développées, même si elles y sont importantes. Elles le sont dans une étude parallèle, publiée en 1997 (Coste, Moore \& Zarate) où la compétence sociale de communication verbale est posée avant tout comme plurielle et non réduite à des échanges dans une seule langue. La nature de la compétence plurilingue et pluriculturelle amène à décrire celle-ci comme un répertoire complexe de compétences en

10. [http://goo.gl/aocsPi] 
différentes langues, une réserve de ressources formelles et sociolinguistiques et un ensemble d'expériences constitutives de la personne. Il revient à l'école d'assurer le développement de cette compétence, qui est actuellement segmentée entre différentes matières scolaires qui s’ignorent, le plus souvent : il convient de rétablir des cohérences entre les différents enseignements de langue (nationale, maternelle, étrangère, classique...). C'est ainsi que la langue de scolarisation entre en lice.

Par la suite, la question de la langue de scolarisation va recevoir encore davantage de poids du fait de la prise de conscience des difficultés de sa maîtrise par les apprenants. C'est dans cette langue que sont impartis les enseignements des matières scolaires. Dans chaque matière scolaire (chimie, histoire, mathématiques...), des formes d'expression (orale et écrite) spécifiques sont nécessaires pour exposer, rendre accessibles, faire acquérir et utiliser les concepts, les procédures et les connaissances qui sont propres à chacune des matières. Or l'acquisition ces formes d'expression ne s'effectue pas de manière spontanée, alors que leur maîtrise convenable est un élément qui détermine la réussite scolaire, comme le montrent les enquêtes PISA (Programme international pour le suivi des acquis des élèves) de ''OCDE $^{11}$ ou PIRLS (Programme international de recherche en lecture scolaire) de l'International Association for the Evaluation of Educative Assesment $(\text { IEA })^{12}$. Les dispositions à prendre dans ce domaine relèvent à l'évidence de la lutte contre l'échec scolaire, problème répandu dans les sociétés européennes, s'il en est. C'est ce qui a conduit le Conseil de l'Europe à attirer l'attention de ses États membres sur cette question, au moyen d'une Recommandation particulière, élaborée au sein de l'Unité des politiques linguistiques $(2014)^{13}$. Car l'enjeu de la maîtrise de ces dimensions linguistiques pour la réussite dans chaque matière est particulièrement visible dans les difficultés scolaires rencontrées par les enfants issus de l'immigration, ceux venant de milieux socioculturels défavorisés ou encore les enfants utilisant d'autres langues dans leur vie quotidienne. Les difficultés de ces groupes d'apprenants sont en fait des révélateurs des obstacles auxquels se trouve confrontée une grande partie des apprenants (Cummins 2010 ; Thürmann, Vollmer \& Pieper 2010).

\section{LA LANGUE DE SCOLARISATION AU CEUR DU DISPOSITIF DU CONSEIL DE L'EUROPE}

Le projet du Conseil relatif aux langues de scolarisation (lancé en 2005), outre la Recommandation à peine citée, tout à fait exceptionnelle sur ce sujet pour une organisation intergouvernementale, a abouti à la Plate-forme pour

\footnotetext{
11. Organisation de coopération et de développement économiques.

12. [http://timss.bc.edu/pirls2016/framework.html]

13. Recommandation $\mathrm{CM} / \operatorname{Rec}(2014) 5$ aux États membres sur l'importance de compétences en langue(s) de scolarisation pour l'équité et la qualité en éducation et pour la réussite scolaire.
} 
l'éducation plurilingue et interculturelle, intitulée "Langues dans l'éducation, langues pour l'éducation », disponible sous forme d'un site dédié (créé en 2009). Celle-ci s'ouvre pleinement aux problématiques relatives aux langues de scolarisation et aux dimensions linguistiques de l'enseignement des connaissances dans les différentes disciplines (histoire, sciences, mathématiques, littérature...), en les reliant clairement à celles relatives aux langues étrangères, régionales, minoritaires ou de la migration ${ }^{14}$. L'Unité des politiques linguistiques a donc procédé dans ce domaine avec les moyens qui sont les siens : des Recommandations (voir supra) et des documents de cadrage partagés.

Mais les questions que pose la langue de scolarisation ne sont pas de même nature, selon qu'on les envisage à travers les matières scolaires ou comme matière scolaire spécifique (le français en France, le grec en Grèce), les premières étant moins politiques que les secondes.

\section{La langue de scolarisation à travers les matières scolaires : apprendre la rhétorique de la connaissance}

C'est sur ce domaine que le Conseil de l'Europe a fait porter ses efforts de sensibilisation des États membres. Les matières scolaires sont enseignées dans la/une langue de scolarisation. Or les communautés scientifiques/technologiques (au sens large), dont les matières scolaires sont l'émanation transposée, ont élaboré des formes spécifiques de communication verbale. De même qu’il y a des "manières de pratiquer " une science ou une technique, il y a des "manières de dire " la science et la technique. Les communautés de spécialistes ont développé, avec le temps, des "règles ", explicitées ou non, qui caractérisent "leur rhétorique ».

Ces « règles pour bien dire » les sciences et les technologies sont aussi présentes à l'école, d'une certaine manière, dans les matières scolaires qui correspondent à ces disciplines. Ces usages sociaux et " culturels " particuliers d'emploi d'une langue donnée, qui concernent surtout les formes écrites et les formes orales officielles ou publiques de la communication, ne se réduisent pas aux règles formelles de la grammaire de ces langues (sur l'ensemble de cette question, voir Beacco, Coste, van de Ven et Vollmer, 2010). Entrer dans l'univers d'une science suppose de saisir ses concepts et ses démarches, mais aussi de s'approprier la rhétorique de cette communauté qui fixe les normes de ses échanges verbaux. Ces formes de la communication verbale des sciences et des technologies (communication orale officielle ou publique, textes écrits) sont bien différentes de la communication quotidienne, orale ou écrite.

14. [http://goo.gl/ZUqbfV] 
Apprendre à gérer des formes de l'expression scientifique n'implique pas uniquement de sensibiliser les enseignants de disciplines à l'intérêt d'activités relatives à l'emploi correct de la terminologie disciplinaire ou à la correction grammaticale et orthographique. Ces activités sont très certainement très utiles, en particulier pour ce qui est de l'emploi d'un registre "surveillé ». Elles ne suffisent cependant pas à faire acquérir la maîtrise, en compréhension et en production, des formes régulées et conventionnelles de la communication dans lesquelles les connaissances se constituent, s'exposent et sont discutées.

Pour inscrire de telles préoccupations dans les programmes d'enseignement, on peut utiliser des descripteurs, à la manière du CECR. Ces indications peuvent se situer au niveau le plus élevé des programmes (finalités) ou constituer un ensemble de référence pour toutes les matières qui sera ensuite décliné par matière. Ainsi, à un descripteur de rang élevé comme : « est capable de construire des textes en utilisant la terminologie adéquate ", peut correspondre, pour les matières relevant des sciences sociales (géographie, histoire...), un descripteur plus ciblé, comme : "peut écrire des textes de sciences sociales en employant la terminologie de manière précise, en mettant en évidence le raisonnement ou les démarches qui conduisent aux conclusions et en utilisant des sources de manière adéquate» (Norwegian Directorate for Education and Training, 2012).

Mais cette spécification est encore trop large et il convient de l'affiner en renvoyant aux genres de textes attendus dans chaque matière (dans l'exemple donné, on ne mentionne que des fonctions cognitives linguistiques isolées), en en décrivant les règles et la structure et en identifiant le matériel linguistique nécessaire, tous éléments qui définissent la rhétorique de la matière concernée au niveau le plus «bas » du programme. À cette fin, on ne saurait faire l'économie d'analyses linguistiques de discours, de genres de textes comme l'article scientifique, le cours universitaire en amphithéâtre, les textes des manuels scolaires, les formes des épreuves écrites ou des travaux écrits attendus dans les matières scolaires... On rappellera que l'analyse du discours a été introduite précisément à cette fin en didactique du français enseigné comme langue étrangère, à partir des années 1970. On en trouvera une utilisation récente pour l'établissement d'objectifs linguistiques pour les programmes d'histoire dans Beacco 2010 ${ }^{15}$. L'ensemble de ces préoccupations a abouti à la réalisation d'un guide spécifique (Beacco et al., 2015).

\section{La langue de scolarisation comme matière scolaire spécifique}

L'enseignement de la matière «langue de l'école » (français, allemand, italien ou romanche en Suisse, polonais en Pologne ${ }^{16}$ ) ne relève pas des mêmes problématiques, même si l'on ne prend pas en considération celle de son statut.

16. Indépendamment de la désignation officielle de ces matières dans les programmes nationaux. 
Produit de longues traditions, ces matières reçoivent des configurations diverses suivant les cultures éducatives et cycles scolaires. De ce fait et parce qu'elles répondent à des finalités de formation différenciées, la création d'un cadre de référence partagé n'est pas aisée à envisager.

Par certaines de leurs composantes, ces matières partagent les traits des autres matières. Chargées d'enseigner (en réception et en production) des formes ordinaires et non ordinaires de la communication sociale (en particulier des formes écrites), elles ont à faire accéder à la « rhétorique » de celles-ci (c'est-à-dire aux conventions qui en définissent le caractère approprié) mais aussi à la rhétorique de la connaissance, responsabilité qu'elle partage avec les autres matières.

Elles ont en propre, dans la plupart des cultures éducatives, de donner aux apprenants une connaissance réflexive de la langue et des textes. Cela suppose la maîtrise de catégories descriptives (nombre, aspect...) et de procédures d'analyse (par exemple par substitution), le recours à des jugements de grammaticalité, la verbalisation des intuitions épilinguistiques, etc. Cette dimension spécifique de la langue comme matière ne doit pas conduire à sous-estimer le rôle du répertoire linguistique individuel des apprenants comme ressources pour des activités grammaticales contrastives ou par comparaison : le détour par des langues autres est de nature à mettre en relief les fonctionnements de la langue de scolarisation.

La matière langue de scolarisation a aussi la responsabilité d'assurer l'accès à la littérature, le plus souvent aux œuvres du patrimoine culturel national, qui présentent une forte valeur identitaire, qu'elles soient limitées au canon littéraire ou qu'elles fassent aussi une place à la littérature contemporaine, aux littératures étrangères ou à la littérature mondialisée, aux "paralittératures populaires » (BD, romans policiers, science-fiction...). Les choix de ces contenus mêmes sont objets de débats internes, tout autant que ceux relatifs aux méthodes pour y accéder : de l'explication de texte « à la française » à la réaction personnelle subjective à un texte.

Cet état des lieux rend complexe l'établissement d'un document-cadre partagé à d'autres niveaux que celui de finalités comme :

" la capacité à adapter ses modes de raisonner et d'argumenter; le développement du sens esthétique et de la sensibilité à l'expression culturelle ; le respect et l'intérêt pour les valeurs d'autrui et une disposition ouverte au pluralisme dans la communication; la collaboration participative à des projets communs; la compréhension de ses propres émotions et de celles d'autrui. Ainsi, les objectifs de la langue comme matière concernent le développement personnel au sens large, la langue comme matière contribuant non seulement à l'acquisition de compétences de communication mais aussi, [...] à l'" apprendre à apprendre ", aux compétences sociales et civiques, à la sensibilité et à l'ouverture culturelles. ${ }^{17}$

17. La langue comme matière : [http://goo.gl/bdBTwr] 
Il semble surtout important de veiller à ce que cet enseignement, tout autant que les autres, permettent de construire des appartenances collectives plurielles et ouvertes à l'altérité, quelle soit culturelle ou linguistique.

Le Conseil de l'Europe est la seule organisation intergouvernementale européenne à avoir focalisé ses efforts de coopération avec les États membres sur la langue de scolarisation. Il aborde cette question dans le cadre holistique de l'éducation plurilingue et interculturelle, en se situant plutôt sur le plan de l'ingénierie des formation que sur le plan purement politique (quelle(s) langue(s) de scolarisation) où il ne peut intervenir, sous forme de recommandations, qu'au nom des droits des minorités. Mais une langue de minorité peut être introduite dans le système éducatif, sous bien des formes, avant d'accéder au statut suprême de langue de scolarisation. Des espaces de négociation existent et des revendications " extrêmes " (devenir langue comme matière) dissimulent souvent mal des projets d'autonomisation, sous la forme d'États souverains distincts et... monolingues.

\section{BiBLIOGRAPHIE}

BEACCO J.-C. (2010) : Histoire: Une démarche et des points de référence - Éléments pour une description des compétences linguistiques en langue de scolarisation nécessaires à l'enseignement/apprentissage de l'histoire (fin de la scolarité obligatoire), Strasbourg, Conseil de l'Europe.

BEACCO J.-C., COSTE D., VAN DE VEN P.-H., VOLLMER H. (2010) : Langue et matières scolaires. Dimensions linguistiques de la construction des connaissances dans les curriculums, Plate-forme de ressources et de références pour une éducation plurilingue et interculturelle, Conseil de l'Europe. [http://goo.gl/uKjQjQ]

BEACCO J.-C., GOULLIER F., FLEMING M., THÜRMAN E., VOLLMER H. (2015) : (version provisoire), Les dimensions linguistiques de toutes les matières scolaires. Un Guide pour l'élaboration des curriculums et pour la formation des enseignants, Strasbourg, Conseil de l'Europe.

COSTE D., MOORE D., ZARATE G. (1997) : Compétence plurilingue et interculturelle, Strasbourg, Conseil de l'Europe.

CUMMINS J. (2010) : De l'importance des données de la recherche empirique pour les politiques éducatives en faveur des apprenants en difficulté, Plate-forme de ressources et de références pour une éducation plurilingue et interculturelle, Conseil de l'Europe. [http://goo.gl/SU0q0W]

Norwegian Directorate for Education and Training (ed.) (2012) : Framework for Basic Skills. To use for subject curricula, Groups appointed by the Norwegian Directorate for Education and Training. [http://goo.gl/vLVJcH] (05/2014).

THÜRMANN E., VOLLMER H., PIEPER I. (2010) : Langue(s) de scolarisation et apprenants vulnérables, Plate-forme de ressources et de références pour une éducation plurilingue et interculturelle, Conseil de l'Europe. [http://goo.gl/vLVJcH] 
